\title{
EVIDENCES FOR COMPLEX FORMATION BETWEEN POLYMYXIN B AND LIPOPOLYSACCHARIDES FROM SERRATIA MARCESCENS*
}

\author{
Joseph C. Tsang**, Douglas A. Weber and David A. Brown \\ Departments of Chemistry and Biological Sciences, Illinois State University \\ Normal, Illinois, U.S.A. \\ (Received for publication January 27, 1976)
}

\begin{abstract}
In vitro and in vivo complex formations of polymyxin B and lipopolysaccharides (LPS) from resistant and sensitive cells of Serratia marcescens were studied by polyacrylamide gel electrophoresis in sodium dodecyl sulfate and electron microscopy. In vitro treatment of LPS from resistant cells with polymyxin B gave two populations of spherical complexes of different molecular weights as determined electrophoretically. Similar treatment of LPS from sensitive cells resulted in dissociation of the LPS-protein and subsequent complexing with the LPS moiety into stable spheres. In vivo treatment of resistant cells with polymyxin B resulted in LPS-polymyxin B complexes which were comparatively smaller and existed in two morphological forms: spheres and linear ribbons. LPS from the sensitive cells were degraded extensively into small rods and an amorphous mass by the in vivo polymyxin B treatment. In both systems, the electrophoretic results consistently matched the electron microscopic evidences for complex formation of LPS with polymyxin B. It is suggested that the disruptive effects of polymyxin B on LPS in the outer membrane of $S$. marcescens may be the explanation for the change in permeability barrier in the resistant cells and disorganization of the outer membrane and subsequent death in the sensitive cells. Furthermore, the ability of the LPS to complex with the polymyxin B molecules in resistant cells may be the basis of their resistance to the antibiotic.
\end{abstract}

Polymyxin B, a cationic polypeptide antibiotic, interacts with various anionic cellular components of gram-negative bacteria such as lipopolysaccharide (LPS), ${ }^{1,2)}$ phospholipids (PL), ${ }^{3 \sim 6)}$ deoxyribonucleic acid (DNA), ${ }^{7 \sim 8)}$ and ribosomes..$^{8-10)}$ Polymyxin $\mathbf{B}$ treatment of LPS isolated from susceptible bacteria results in a breakdown of the LPS molecule (in vitro effect). ${ }^{11}$ When whole cells are treated with the antibiotic, blebs are formed on the outer membrane of the cell envelope $\mathrm{e}^{7,12 \sim 14)}$ and subsequently, disorganization of the outer membrane occurs if the cells are susceptible to the antibiotic. Although the formation of the blebs in the outer membrane has been suggested to be caused by an aggregation of the antibiotic with outer membrane components such as LPS and $\mathrm{PL}^{9)}$ (in vivo effect), direct evidences for the formation of such aggregates or complexes have not been shown, nor was the sequence of events from the moment of complex formation to the disorganization of the outer membrane described. In this communication, we report the electrophoretic and electron microscopic evidences of the in vitro and in vivo formation of complexes between polymyxin B and LPS from cells of Serratia marcescens, which were either resistant or sensitive to the antibiotic.

* Preliminary accounts of this investigation was presented at the 11th Midwest Regional Meeting of American Chemical Society, October 30 31, 1975, Southern Illinois University, Carbondale, Illinois, U.S. A.

** To whom all correspondence should be addressed: Dr. JosepH C. TsANG, Department of Chemistry, Illinois State University, Normal, Illinois 61761, U.S. A. 


\section{Experimental}

The bacterial cultures consisted of two nonclinical strains, 08 (polymyxin B resistant) and Bizio (polymyxin B sensitive) and two clinical isolates, 6292 (polymyxin B resistant) and 13378 (polymyxin B sensitive). The clinical isolates were supplied by Dr. M. A. Miller of Peoria School of Medicine, University of Illinois College of Medicine (U.S. A.). All strains were grown in an enriched medium of casamino acids as previously described, $\left.{ }^{15},{ }^{18}\right)$ with aeration at room temperature and harvested after 17 19 hours of growth. Cells harvested from 1 liter of medium were treated with $20 \mathrm{mg}$ of polymyxin B sulfate (Burroughs Wellcome) in $75 \mathrm{ml}$ of $0.9 \%$ saline at $\mathrm{pH} 7.3$ for 1 minute at $37^{\circ} \mathrm{C}$ according to the method of CERNY and Teuber. ${ }^{17)}$

Lipopolysaccharide was extracted from polymyxin B treated and untreated cells of the resistant and sensitive strains of $S$. marcescens by the modified hot aqueous phenol method of WeSTPHAL as previously described. ${ }^{16)}$ In vitro complex formation of polymyxin B with LPS was carried out by adding $1 \mathrm{mg}$ of LPS form either resistant or sensitive cells to $0.2 \mathrm{ml} 0.9 \%$ saline solution containing $0.05 \mathrm{mg}$ of polymyxin $\mathrm{B}, \mathrm{pH} 7.3$. After mixing for 1 minute at $37^{\circ} \mathrm{C}$, the LPS-polymyxin B solution was either placed on a carbon-coated Formvar grid for electron-microscopy or added to a solubilizing solvent for polyacrylamide gel electrophoresis (PGE) in sodium dodecyl sulfate (SDS).

Electron-microscopy of LPS was performed using the negative staining technique. A drop of the LPS solution or LPS-polymyxin B mixture was placed on a carbon coated Formvar grid and stained with $1 \%$ phosphotungstic acid, $\mathrm{pH}$ 6.8. The negatively stained material was examined in an Hitachi HU-11 A Electron Microscope at an accelerating voltage of $50 \mathrm{kV}$.

Polyacrylamide gel electrophoresis in SDS of LPS preparations from resistant and sensitive strains before and after treatment with polymyxin B, as well as isolated LPS after complexing with polymyxin B was carried out in a $5.25 \%$ gel containing $0.5 \%$ SDS in a $0.1 \mathrm{~m}$ phosphate buffer, pH 7.1 for $2 \frac{1}{2}$ hours at $8 \mathrm{~mA} /$ tube. Duplicate gels were stained for protein with coomassie blue, and LPS with a "stains-all" carbocyanine dye.17) All stained gels were scanned on a Beckman ACTA VI Spectrophotometer at $462 \mathrm{~nm}$ for gels stained for LPS, at $600 \mathrm{~nm}$ for gels

Fig. 1. Electrophoretic patterns of polymyxin B, isolated LPS, and LPS-polymyxin B complexes of Serratia marcescens.

(a) Polymyxin B; (b) LPS from strain 08; (c) LPS from strain 08 after in vitro treatment with polymyxin B; (d) LPS from strain Bizio; and (e) LPS from strain Bizio after in vitro treatment with polymyxin B.

Samples were dissolved in a solvent made up of $2.0 \%$ SDS, $1.0 \%$ 2-mercaptoethanol, $10 \%$ glycerol in $10 \mathrm{~mm}$ phosphate buffer, $\mathrm{pH}$ 7.1. $5.25 \%$ Acrylamide gel in $0.05 \mathrm{M}$ phosphate buffer, pH 7.1 was used.

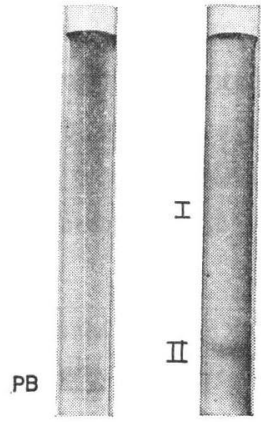

(a)

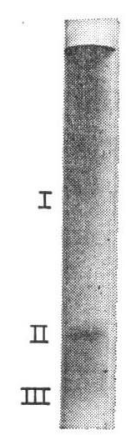

(c)

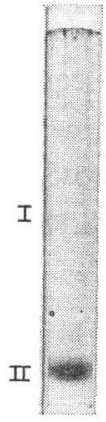

(d)

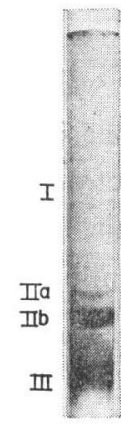

(e) stained for protein, and at $280 \mathrm{~nm}$ for unstained gels to detect proteinous material, including polymyxin B. Estimation of the molecular weights of the separated components was performed according to standard methods.

\section{Results}

Polyacrylamide Gel Electrophoresis of Various LPS Preparations

In order to determine the chemical and physical natures of the LPS components in the preparations after various treatments, PGE along with differential staining techniques were used. Fig. 1 shows the electrophoretic patterns of polymyxin B, isolated LPS, and LPS treated with polymyxin B from two strains of $S$. marcescens. Before staining, polymyxin $\mathrm{B}$ gave a component which absorbed strongly at $280 \mathrm{~nm}$. This component (PB) stained positively for protein and had an apparent molecular weight of 15,000 daltons (Fig. 1-a). Since the 
actual molecular weight of polymyxin B itself is only 1213, it is likely that this component represents a micelle of polymyxin B with SDS in the gel. The gel patterns of LPS isolated from strain 08 and strain Bizio are shown in Fig. 1-b and 1-d, respectively. In both cases there were two detectable components. The major one (II) in strain 08 had a molecular weight of approximately 18,500 daltons while the similar one in strain Bizio had a slightly lower molecular weight of 17,000 daltons. These components stained positively for both LPS and protein. After treatment with polymyxin B, LPS (08) gave three components (Fig. 1-c) with molecular weights of 62,500 (I), 28,000 (II) and 16,000 (III) daltons. Similar treatment of LPS from the sensitive strain Bizio gave a complex pattern with multiple peaks (Fig. 1-e). In the regions stained positively for LPS (Figs. 2-g and 2-a), a broad band (I) with multiple small peaks was found in the molecular weight range of 47,000 67,000 daltons. In addition, a sharp band (II b) of molecular weight of 27,500 daltons was also found. When the gels were stained for protein, multiple peaks were again found in the region of the broad band $\mathbf{I}$, as well as in a lower molecular weight region of 22,500 25,500 daltons. Components II a and III (Fig. 1-e; Fig. 2-g and 2-h) stained positively for protein but not LPS. The gel scans (Figs. 2-i and $2-j$ ) of LPS components in preparations from the resistant cells treated with polymyxin B were similar to those of the untreated LPS from 08 strain (Fig. 2-a). Table 1 summarizes the apparent molecular weights of the separated components from various LPS fractions. In the in vitro treated samples, the increases $(54 \%$ $56 \%$ ) in the apparent molecular weight of the major components (II) from LPS fractions of the resistant cells (08 and 6292) were slightly lower than those $(59 \sim 62 \%)$ of the LPS fractions from sensitive cells (Bizio and 13378). The increases $(6 \sim 13 \%)$ in the components of the LPS after in vivo treatment of the resistant cells were significantly lower in comparison with those after in vitro treatment $(54 \sim 56 \%)$. Thus, it appears that the LPS components in the outer membrane were protected from interacting with the antibiotic during the in vivo treatment.
Fig. 2. Gel scans of various electrophoresed LPS preparations from resistant and sensitive strains of Serratia marcescens.

LPS from resistant strains 08 (a) and 6292 (b) and sensitive strains Bizio (e) and 13378 (f); their corresponding polymyxin B complexes are given in (c), (d), and (g), (f), respectively. LPS isolated from polymyxin $\mathrm{B}$ treated resistant cells of 08 (i) and 6292 (j) are also included.

Gels were scanned at $600 \mathrm{~nm}$ for protein stained components (-) and $462 \mathrm{~nm}$ for LPS containing materials (-..-).

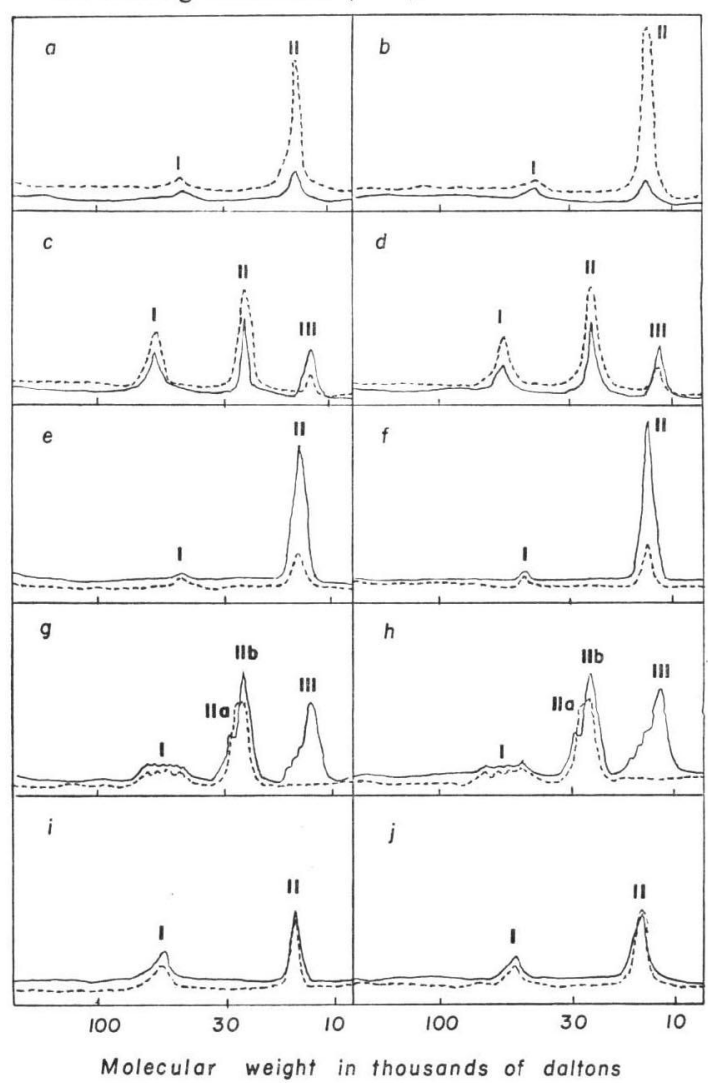


Table 1. Major polymyxin-lipopolysaccharide complexes in LPS preparations from resistant and sensitive strains of $S$. marcescens

\begin{tabular}{|c|c|c|c|c|}
\hline \multicolumn{2}{|c|}{ Fractions } & \multirow{2}{*}{$\begin{array}{c}\text { Apparent } \\
\text { Mol. Wts. } \\
62,500 \\
28,000 \\
16,000\end{array}$} & \multirow{2}{*}{$\begin{array}{c}\text { Increase in } \\
\text { Mol. Wts. } \\
9,500^{\mathrm{a}} \\
10,000^{\mathrm{a}} \\
1,000^{\mathrm{b}}\end{array}$} & \multirow{2}{*}{$\begin{array}{c}\% \text { Increase } \\
17.9 \\
56.6 \\
6.7\end{array}$} \\
\hline Polymyxin-LPS (08) & $\begin{array}{l}\text { Component I } \\
\text { Component II } \\
\text { Component III }\end{array}$ & & & \\
\hline Polymyxin-LPS (6292) & $\begin{array}{l}\text { Component I } \\
\text { Component II } \\
\text { Component III }\end{array}$ & $\begin{array}{l}58,000 \\
28,000 \\
16,500\end{array}$ & $\begin{array}{l}6,500 \\
9,500 \\
1,500\end{array}$ & $\begin{array}{l}12.6 \\
54.4 \\
10.0\end{array}$ \\
\hline Polymyxin-LPS (Bizio) & $\begin{array}{l}\text { Component I } \\
\text { Component II-b } \\
\text { Component III }\end{array}$ & $\begin{array}{l}55,500^{c} \\
27,500 \\
15,500\end{array}$ & $\begin{array}{r}4,000^{\mathrm{a}} \\
10,500^{\mathrm{a}} \\
500^{\mathrm{b}}\end{array}$ & $\begin{array}{r}7.8 \\
61.8 \\
3.3\end{array}$ \\
\hline Polymyxin-LPS (13378) & $\begin{array}{l}\text { Component I } \\
\text { Component II-b } \\
\text { Component III }\end{array}$ & $\begin{array}{l}54,000^{\mathrm{c}} \\
27,000 \\
15,500\end{array}$ & $\begin{array}{r}2,500^{\mathrm{a}} \\
10,500^{\mathrm{a}} \\
500^{\mathrm{b}}\end{array}$ & $\begin{array}{r}4.8 \\
58.8 \\
3.3\end{array}$ \\
\hline LPS (08)-Treated Cells & $\begin{array}{l}\text { Component I } \\
\text { Component II }\end{array}$ & $\begin{array}{l}57,000 \\
17,000\end{array}$ & $\begin{array}{r}5,000^{\mathrm{a}} \\
11,000^{\mathrm{a}}\end{array}$ & $\begin{array}{l}9.4 \\
5.6\end{array}$ \\
\hline LPS (6292)-Treated Cells & $\begin{array}{l}\text { Component I } \\
\text { Component II }\end{array}$ & $\begin{array}{l}53,500 \\
21,000\end{array}$ & $\begin{array}{l}2,000^{\mathrm{a}} \\
2,500^{\mathrm{a}}\end{array}$ & $\begin{array}{r}3.9 \\
13.5\end{array}$ \\
\hline
\end{tabular}

a amount increase based on the molecular weights of the corresponding LPS components in preparations before treatment with polymyxin B

$\mathrm{b}$ amount increase based on the molecular weights of polymyxin B after electrophoresis

c present as a broad band with a molecular weight range from 47,000 to 67,000 daltons

\section{Determination of Protein/LPS Ratio}

In order to determine the relative amount of polymyxin B complexing with the LPS components, the ratio of the maximum absorbance of the protein stained components to that stained for LPS in the gels was calculated (Table 2). By this method, an increase in the ratio in the component would indicate binding of polymyxin B to the LPS. On the other hand, a decrease of this ratio could be interpreted as an indication for dissociation and/or degradation of the LPS-protein complex. When the ratios of the various components in the preparations from the resistant cells are compared, it can be seen that the amount of increase after the in vivo treatment (0.6) parallels those after the in vitro treatment $(0.5 \sim 0.7)$. This seems to indicate that equal amounts of polymyxin may be binding to the major components of LPS

Table 2. Protein/LPS ratio of component II in various LPS preparations of $S$. marcescens

\begin{tabular}{c|l|c|c|c|c|c}
\hline \multicolumn{2}{c|}{ Strains } & $\begin{array}{c}\text { Before } \\
\text { polymyxin B } \\
\text { treatment }\end{array}$ & $\begin{array}{c}\text { After in vivo } \\
\text { treatment }\end{array}$ & $\begin{array}{c}\text { After in vitro } \\
\text { treatment }\end{array}$ & $\begin{array}{c}\text { Increase after } \\
\text { in vivo } \\
\text { treatment }\end{array}$ & $\begin{array}{c}\text { Increase after } \\
\text { in vitro } \\
\text { treatment }\end{array}$ \\
\hline \multirow{2}{*}{ Resistant } & 08 & 0.48 & 1.35 & 0.88 & 0.87 & 0.50 \\
& 6292 & 0.15 & 0.83 & 0.90 & 0.68 & 0.75 \\
\hline \multirow{3}{*}{ Sensitive } & Bizio & 4.24 & N. A. & 1.26 & - & -2.98 \\
& 13378 & 4.10 & N. A. & 1.54 & - & -2.56 \\
\hline
\end{tabular}

\footnotetext{
${ }^{a}$ N. A. = not available
} 
Fig. 3. Morphological appearance of LPS and their polymyxin B complexes of resistant and sensitive strains of Serratia marcescens.

(a) LPS from resistant strain 08 before polymyxin B treatment

(b) LPS from resistant strain 08 after in vitro polymyxin B treatment

(c) LPS from resistant strain 08 after in vivo polymyxin B treatment

(d) LPS from sensitive strain Bizio before polymyxin B treatment

(e) LPS from sensitive strain Bizio after in vitro polymyxin B treatment

(f) LPS from sensitive strain Bizio after in vivo polymyxin B treatment

In the untreated LPS, note the crescent-edged spheres (arrow); many spheres from the sensitive strain Bizio did not have complete peripheries (arrow). After the in vitro treatment, two populations of spheres (arrows) were present in those of the resistant strain 08 while a few surviving spheres existed among the numerous rods from the sensitive strain Bizio (arrow). From the in vivo treatment, note the smaller spheres and linear forms from the 08 cells (arrow) and the occasional rods in the amorphous mass of the Bizio (arrow). The marker bar in all micrographs represents $0.5 \mu$.
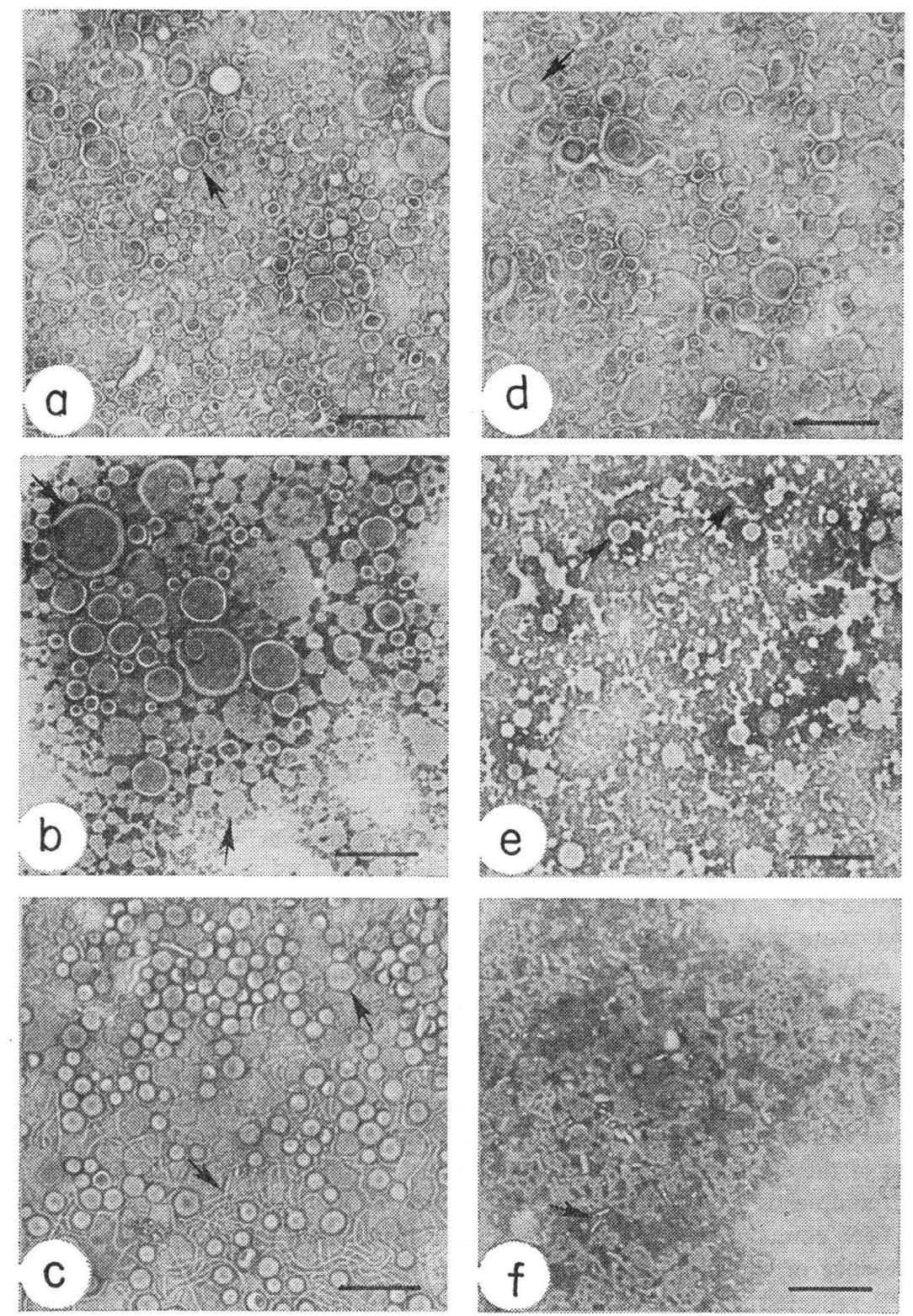
of the resistant cells regardless of treatment. A similar comparison was not possible with samples from the sensitive cells because of the lack of recoverable LPS after the in vivo treatment of the cells. However, when the ratios of protein to LPS content of the untreated samples $(4.10 \sim 4.24)$, are compared with those after in vitro treatment $(1.26 \sim 1.54)$, it appears that extensive degradation of the LPS-protein complexes in the preparation of the sensitive cells occurred.

\section{Electron-microscopic Characterization of Various LPS Fractions}

Figs. 3-a and 3-d show the normal morphology of LPS isolated from untreated whole cells of the polymyxin B resistant strain 08 and the polymyxin B sensitive strain Bizio, respectively. LPS from these cells displayed the spherical crescent-edged spheres similar to those described for the normal morphology of LPS from Salmonella. ${ }^{21)}$ LPS isolated from resistant strains (08 and 6292) after the in vitro polymyxin B treatment (LPS+Polymyxin B) of the preparations (Fig. 3-b) retained some of the spherical characteristics of the untreated LPS, but the spheres seemed to be either larger and more uniform or smaller and less distinct in their peripheries. The general morphology of LPS from the sensitive strains (Bizio and 13378), resembled those of the resistant strains, but the globules often seemed to form incomplete spheres with irregular peripheries (Fig. 3-d). After the in vitro polymyxin B treatment, only a few spheres remained (Fig. 3-e), with occasional short rods present. The effect of polymyxin B in vitro, on the LPS from the sensitive strains of $S$. marcescens was similar to that on LPS from E. coli. ${ }^{11}$ LPS isolated from resistant cells after they were treated with polymyxin B (in vivo treatment) showed a modification of the morphology similar to those of the in vitro treatment. In addition numerous linear forms were present (Fig. 3-c). However, LPS from sensitive cells treated in vivo with polymyxin B was degraded into many short rods and a general amorphous mass (Fig. 3-f). This supplements the previous findings that LPS in the outer membrane in these treated cells was degraded so extensively that little LPS was recoverable by normal extraction procedures for other characterizations (unpublished results).

\section{Discussion}

It has been reported that in various in vitro systems, polymyxin B (PB) could break down isolated LPS ${ }^{11)}$ and inactivate endotoxin (LPS-protein complex). ${ }^{19,20)}$ The effect of PB on the LPS and/or outer membrane has been suggested to be caused by the interaction of the lipophilic and lipophobic groups of the antibiotic and the LPS molecules. ${ }^{1,5,6)}$ These interactions may alter the numerous $\mathrm{pH}$ dependent morphological forms of LPS, such as droplets and ribbons ${ }^{22}$ as well as globules, ${ }^{21)}$ slender rods, ${ }^{23)}$ flat twisted discs, ${ }^{24)}$ and branched ribbons. ${ }^{25)}$ By electron microscopy we were able to observe the changes in the normal morphology (the spherical form) of the LPS from $S$. marcescens after the in vivo treatment with PB. By polyacrylamide gel electrophoresis in SDS, complexes of different sizes were resolved and separated. When the LPS fractions from the resistant cells were treated with $\mathrm{PB}$, three discrete components with molecular weights of $60,000,28,000$ and 16,000 daltons were obtained. The first two were shown to be polymyxin B-LPS complexes by their increased molecular weight as well as by their increased protein/LPS ratio (Table 2). The third component had an apparent molecular weight similar to that of the electrophoresed PB. The ability of the LPS components from the resistant cells to form complexes with $\mathrm{PB}$, indicated that $\mathrm{PB}$ not only modified, but also increased the size of the LPS components (Fig. 3-b), and had little effect on their stability. On the other hand, the presence of the multiple components in the reaction mixture of LPS from sensitive strains and PB reflected significant degradation of the LPS components by the antibiotic. The 
large decrease of the protein/LPS ratio from 4.10 to 1.25 clearly indicated that disruption occurred between the protein LPS linkages. Morphologically, these multiple components were assumed to be present as non-resolvable particles (Fig. 3-e), while the surviving spheres were present as the 27,500 daltons complex (Fig. 2-g). Similarly, the two populations of spheres (Fig. 3-c) observed in the in vitro treatment of LPS fractions from resistant cells corresponded to the complex formed in the electrophoretic system, namely, the 60,000 daltons and the 27,500 daltons components (Fig. 2-c and 2-d). Despite the presence of various linkages in the LPS-protein complex which may be sensitive to phenol extraction ${ }^{16,26)}$ and mild acetic acid treatment, ${ }^{27)}$ they may be protected in their native state in the outer membrane ${ }^{21)}$ during phenol extraction. Bonds untouched by phenol treatment may not survive the interaction with PB. Also, the in vivo treatment with PB on the LPS-protein complexes in the cell envelope prior to phenol treatment may not be any less damaging. Indeed, after in vivo treatment, the LPS components in strain Bizio were degraded to such extent (Fig. 3-f) that few complete spheres survived. Subsequent phenol extraction failed to recover any significant amount of LPS. Several explanations may be possible for the resulting dissociation of the LPS-protein complex by PB. First, LPS preparations from the phenol-water method may contain various amounts of protein in association with the LPS components. Treatment with PB could release a heterogeneous population of protein components and the LPS component would then be available for complex formation with PB. The multiple protein bands in the molecular weight region of 22,500 25,500 daltons as well as the 32,000 daltons component support this idea (Fig. 3-g). The second possible explanation would be that LPS components attaching to the protein moiety of the LPS-protein entity are heterogeneous in nature. In the presence of competing PB molecules, some of the heterogeneous LPS components would be released or displaced by binding to PB while the other remained in association with the protein components. In light of the previously reported heterogeneity of LPS in $S$. marcescens, ${ }^{2 \theta)}$ the second explanation seems to be quite attractive.

In conclusion we have provided direct electrophoretic evidence for the formation of complexes between LPS components and PB. Concomitant with the complex formation, electron microscopic results indicated that PB has a considerable effect on the morphology of LPS molecules. The difference in the disruptive effect of PB on LPS in cells of $S$. marcescens with different antibiotic susceptibility might explain the various degrees of damage inflicted on the outer membrane of the cell envelope of the bacteria. In the resistant cells the formation of a complex between LPS and PB might give rise to the morphological alterations of the cell envelope such as bleb formation and the possibility of a change in the permeability barrier. In the sensitive cells, such interactions may be of a transient nature, but the dissociation of the LPS-protein entities as well as degradation of the LPS itself may disorganize the outer membrane with a subsequent increase of antibiotic susceptibility.

\section{Acknowledgement}

The authors express deep appreciation for the financial support for this investigation from the Brown-Hazen Fund of Research Corporation, U.S. A.

\section{References}

1) BADer, J. \& M. Teuber: Action of polymyxin B on bacterial membranes. I. Binding to the O-antigenic lipopolysaccharide on Salmonella typhimurium. Z. Naturforsch. 28c: $422 \sim 430,1973$

2) Rifkind, D.: Studies on the interaction between endotoxin and polymyxins. J. Infect. Dis. 177: 433 438, 1967

3) Hsuchen, C.C. \& D. S. Feingold: Locus of divalent cation inhibition of the bactericidal action of polymyxin B. Antimicr. Agents \& Chemoth. 2: 331 338, 1972

4) Imai, M.; K. Inoue \& S. Nojima: Effect of polymyxin B on liposomal membranes derived from Escherichia coli lipids. Biochim. Biophys. Acta 375: 130 137, 1975

5) Teuber, M. \& J. BADeR: Interaction of polymyxin with the outer membrane of Salmonella 
typhimurium binding to isolated lipopolysaccharides and lipid A. Abst. Com. Meet. Feb. Europ. Biochem. Soc. 8: 26, 1972

6) Teuber, M.: Action of polymyxin B on bacterial membranes. II. Formation of lipophilic complexes with phosphatidic acid and phosphatidylglycerol. Z. Naturforsch. Teil 26: 476 477, 1973

7) Handley, P.S.; L.B. Quesnel \& M. Sturgiss: Ultrastructural changes produced in Proteus vulgaris by synergistic combination of colistin and sulphadiazine. Microbios 10: 211 233, 1974

8) Nakajima, K. \& J. Kawamata: Studies on the mechanisms of action of colistin. I. Formation of an insoluble complex with nucleic acids. Biken J. 8: 225 231, 1965

9) Schindler, P.R. G. \& N. Teuber: Action of polymyxin B on bacterial membranes: Morphological changes in the cytoplasm and in the outer membrane of Salmonella typhimurium and Escherichia coli. Antimicr. Agents \& Chemoth. 8: 95 104, 1975

10) Nakajima, K. \& J. Kawamata: Studies on the mechanisms of action of colistin. III. Precipitation of Escherichia coli ribosome with colistin. Biken J. 9: 45 50, 1966

11) Lopes, J. \& W.E. InNiss: Electron microscopy of effects of polymyxin on Escherichia coli lipopolysaccharide. J. Bact. 100: 1128 1130, 1969

12) Suganuma, S.; K. Hara, T. Kishida, K. Nakajima \& J. Kawamata: Cytological changes of Escherichia coli caused by polymyxin B. Biken J. 11: 149 155, 1968

13) Wahn, K.; G. Lutsch, T. Rokstroh \& K. ZAPF: Morphological and physiological investigations on the action of polymyxin B on Escherichia coli. Arch. Mikrobiol. 63: 103 116, 1968

14) Koike, M.; K. IIda \& T. Matsuo: Electron microscopic studies on some Proteus strains. Acta Path. Microbiol. Scand. 80: 641 649, 1969

15) Alaupovic, P.; A. C. Olson \& J. C. Tsang: Studies on the characterization of lipopolysaccharide from two strains of Serratia marcescens. Ann. N. Y. Acad. Sci. 133: 546 565, 1966

16) Tsang, J.C.; S.C. Wang \& P. Alaupovic: Degradative effects of phenol on endotoxin and lipopolysaccharide preparations from Serratia marcescens. J. Bact. 117: 786 795, 1974

17) Cerny, B. \& M. Teuber: Comparative polyacrylamide electrophoresis of periplasmic proteins released from gram-negative bacteria by polymyxin B. Arch. Mikrobiol. 82: 361 370, 1972

18) Green, M. R. \& J. V. PAStewkA: Identification of sialic acid-rich glycoproteins on polyacrylamide gels. Anal. Biochem. 65: 66 72, 1975

19) Cooperstock, M. S.: Inactivation of endotoxin by polymyxin B. Antimicr. Agents \& Chemoth. 6: $422 \sim 425,1974$

20) Craig, W. A.; J. H. Turner \& C. M. Kunin: Prevention of the generalized Schwartzman reaction to endotoxin lethality by polymyxin B localized in tissues. Infect. Immun. 10: 287 292, 1974

21) Rothfield, L. \& R. W. Horne: Reassociation of purified lipopolysaccharide and phospholipid of the bacterial cell envelope: Electron microscopic and monolayer studies. J. Bact. 93: 1705 1721, 1967

22) Schramm, G.; O. Westphal \& O. Luderitz: Uber bakterielle Reizstaffe. III. Physikalischchemisches Verhalten eines hochereinigten Colipyrogens. Z. Naturforsch. 76: 594 598, 1952

23) Beer, H.; A.I. Braude \& C. C. Brinton, Jr.: A study of particle sizes, shapes and toxicities present in a Boivin-type endotoxic preparation. Ann. N. Y. Acad. Sci. 133: 450 475, 1966

24) Bladen, H. A. \& S. E. Merganhagen: Ultrastructure of Veilonella and morphological correlation of an outer membrane with particles associated with endotoxic activity. J. Bact. 88: 1482 1492, 1964

25) Shands, J. W.; J. A. Graham \& K. Nath: The morphologic structure of isolated lipopolysaccharide. J. Mol. Biol. 25: 15 21, 1967

26) Wober, W. \& P. Alaupovic: Studies on the protein moiety of endotoxin from gram-negative bacteria. Characterization of the protein moiety isolated by phenol treatment of endotoxin from Serratia marcescens 08 and Escherichia coli 0141: K 85(B). Eur. J. Biochem. 19: 340 356, 1973

27) Wober, W. \& P. Alaupovic: Studies on the protein moiety of endotoxin from gram-negative bacteria. Characterization of the protein moiety isolated by acetic acid hydrolysis of endotoxin from Serratia marcescens 08. Eur. J. Biochem. 19: 357 367, 1973

28) Nowotny, A.; K. R. Cudy, N. L. Neale, A. M. Nowotny, R. Radvany, S. P. Thomas \& D. J. TRIPOLI: Relation of structure to function in bacterial O-antigens. IV. Fractionation of the components. Ann. N. Y. Acad. Sci. 133: 586 603, 1966 